\title{
A Dynamic Compact Model for Ferroelectric Capacitance
}

\author{
Ning Feng, Chang Su, Hao Li, Lining Zhang, Senior Member, IEEE, Qianqian Huang, \\ Runsheng Wang, and Ru Huang, Fellow, IEEE
}

\begin{abstract}
A non-quasi-static model for ferroelectric capacitance is developed in this letter. A state transition in the voltage and time domains between two polarization states is formulated first. The quasi-static model is derived from the state transition of voltage domain, and supports the minor loops. Different from the Preisach model, an initial state is supported, and the modulated coercive voltages are responsible for minor loops. The non-quasi -static model is then derived with the state transition in the time domain, similar to a relaxation approximation in MOSFET modeling. The non-quasi-static model reproduces the saturation loop, minor loops, the frequency-dependent characteristics of measured ferroelectric capacitances, with their origins explained from polarization switching relaxation. The pulse width dependent switching is well reproduced with the model.
\end{abstract}

Index Terms-Ferroelectric, polarization switching, Preisach model, non-quasi-static model.

\section{INTRODUCTION}

Ferroelectric (FE) materials-based electronic devices are attracting wide attentions in the community, for applications in FE random access memory (FeRAM) [1-2] and FE field-effect transistors (FeFET) [3-5], etc. Applications in neuromorphic computing are also emerging utilizing the gradual programing of FE polarization states [6-9]. Besides of the device fabrication, there are also long-term efforts in understanding the switching dynamic of ferroelectric devices [10-12].

One widely used model for ferroelectric polarization is the Preisach model [13-16]. It is a macro-polarization model without going into details of ferroelectric domains, to avoid the complex mathematics. Successful applications of the Preisach model include reproductions of polarization-voltage character -istics, the history-dependence and minor loops. On the other hand, the linear scaling calculation [10] for the minor loops is more from the math consideration. The dynamic switching is also described in a behavioral manner, by a delay term in the voltages $[14,16]$.

This work was supported in part by the Natural Science Foundation of China under grant of 62074006, and in part by the Shenzhen Science and Technology Project under grant of GXWD20200827114656001 and 111 Project (B18001).

Ning Feng, Hao Li and Lining Zhang are with School of Electronic and Computer Engineering, Peking University, Shenzhen, 518055, China. (Email: lnzhang@ieee.org)

Chang Su, Qianqian Huang, Runsheng Wang and Ru Huang are with Institute of Microelectronics, Peking University, 100871, Beijing, China.

In this work a non-quasi-static model is formulated for the ferroelectric polarization switching. It is also based on the macro-polarization approximation; however, the formulations are derived from the rate equations for state transition. Based on the alternative framework, the non-quasi-static model is used to reproduce the saturation loop, minor loops and the polarization switching dynamics.

\section{The StATE TrAnsition FrAMEWORK}

A state transition framework similar to that for phase change memory [17] with the rate equations is the starting point of the developed model. Fig. 1 shows the state transition in the time domain and the voltage domain. $P_{U}$ is the fraction or occupation probability of the upwards polarization, and $P_{D}$ is the counterpart for downwards polarization state. Similar to the Preisach model, only the vertical polarization, e.g. either the upwards or downwards polarization, is considered hence the sum of $P_{U}$ and $P_{D}$ is one. It is the multidomain that leads to the fractional polarization state. The total polarization charge $P$ is the superimposition of charges contributed by the two states.

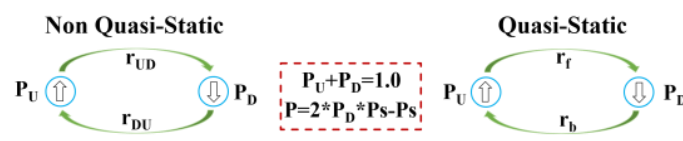

(a)



Fig.1. Two states, the upwards and downwards polarizations, with their fraction $P_{U}$ and $P_{D}$ are defined. (a) The state transition in the time domain is defined by two transition rates depending on the applied voltage, and (b) the transition in the voltage domain is defined by a Fermi-type function.

The state transition happens in both the time domain and the voltage domain. In the time domain, the transition rate from $P_{U}$ to $P_{D}$ is $r_{U D}$. It depends on the nucleation dynamics, however, at present a simple rate is used with the reverse of switching time $t_{s w}[18]$. When the external voltage is positive, the transition from the upwards to downwards state is dominant and the reversal rate $r_{D U}$ is a smaller value $\varepsilon$ [12]. The opposite is true when the external voltage is negative. In the voltage domain, an analogy forward transition to the $P_{D}$ state is given by $r_{f}$, similar to the Fermi-Dirac (FD) statistics [19]. $V_{c}$ is the coercive voltage (similar to the Fermi level), and $V_{0}$ is the domain distribution parameter (similar to the thermal voltage in FD statistics). Similarly, a backward transition $r_{b}$ describes the cases when the applied voltage is decreasing. 


\section{A QuASI-STATIC FerRoelectric Model}

A quasi-static model in electric equilibrium is derived by considering the state transition in the voltage domain, leading to equilibrium state fractions. The ferroelectric polarization does not explicitly depend on the time. The forward transition with increasing voltage is given by the differential equation:

$$
\frac{d P_{D}}{d V_{f e} / V_{0}}=P_{U} r_{f}
$$

and the backward transition is given by:

$$
\frac{d P_{D}}{d V_{f e} / V_{0}}=-P_{D} r_{b}
$$

Eq. (1) is integrated analytically with an assumed initial state (an applied voltage $V_{f e, i}$, and a downwards polarization $P_{D, i}$ ). The solution is given by:

$$
P_{U}=P_{U, i} \frac{1+\exp \left[\left(V_{f e, i}-V_{c}\right) / V_{0}\right]}{1+\exp \left[\left(V_{f e}-V_{c}\right) / V_{0}\right]}
$$

The initial state can be any state within the saturation loop. In a practical case it is also true without exactly knowing the ferroelectric polarization state, and a few wake-up cycles set the ferroelectric saturation loop. Similarly, solving Eq. (2) leads to an analytical equation:

$$
\text { - } P_{D}=P_{D, i} \frac{1+\exp \left[-\left(V_{f e, i}+V_{c}\right) / V_{0}\right]}{1+\exp \left[-\left(V_{f e}+V_{c}\right) / V_{0}\right]}
$$

When the applied voltage changes its direction, e.g., from increasing to decreasing, a new initial state is created. For the saturation loop, Eq. (4) is used after $V_{f e}$ retreats from its peak value, with the initial state of $P_{D, i}=1.0$.

The total charge in the ferroelectric capacitance is obtained with contributions from a linear capacitor $C_{f e}$ :

$$
P_{f e}=\left(2 P_{D}-1\right) \cdot P_{S}+C_{f e} \cdot V_{f e}
$$

where $C_{f e}$ follows its traditional definition. The displacement current in the quasi-static case is calculated by:

$$
i=\frac{d P_{f e}}{d V_{f e}} \frac{d V_{f e}}{d t}
$$

Fig.2(a) plots the polarization-voltage (P-V) curves from the above quasi-static model. Three different initial states are used in the model, and the ferroelectric polarization converge after going through the wake-up process. The obtained saturation loop agrees nicely to that of measured ferroelectric HZO capacitors [20]. Fig.2(b) plots the current density in a ferroelectric capacitor with a triangular voltage waveform of small enough frequency. The current peaks around the coercive voltage as the derivative of polarization over voltage is largest, and changes its sign when the voltage retreats from its peak.

The turning points are defined with the classical protocol [13]. When a new turning point is formed, the trajectory of $\mathrm{P}-\mathrm{Vis}$ immediately defined as it always passes through the turning points stored in the memory. Assuming the new turning point is $\left(V_{f e, i}, P_{D, i}\right)$ and the previous turning point to define the
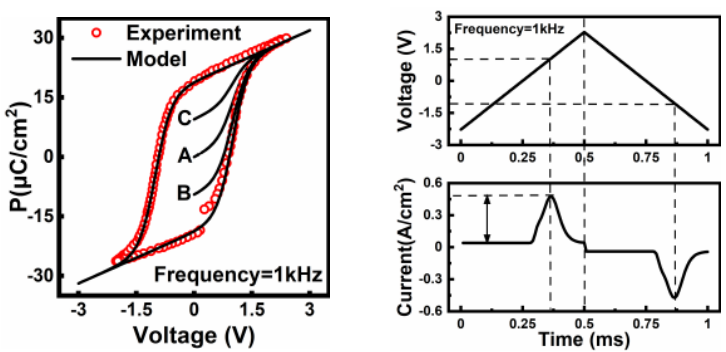

Fig.2. The quasi-static model is verified to reproduce the measurement data of ferroelectric capacitor. (a) The model supports an initial state within the saturation loop, and (b) the quasi-static current model calculates the displacement current for device integration.

trajectory is $\left(V_{f e, t}, P_{D, t}\right)$, both of them are the solution of Eq. (3), with a new coercive voltage $\left(V_{c, i}\right)$ to be determined.

$$
\frac{1-P_{D, t}}{1-P_{D, i}}=\frac{1+\exp \left[\left(V_{f e, i}-V_{c, i}\right) / V_{0}\right]}{1+\exp \left[\left(V_{f e, t}-V_{c, i}\right) / V_{0}\right]}
$$

The solution of $V_{c, i}$ is analytically obtained:

$$
V_{c, i}=V_{0} \cdot \log \left[\frac{P_{U, t} \exp \left(V_{f e, t} / V_{0}\right)-P_{U, i} \exp \left(V_{f e, i} / V_{0}\right)}{P_{D, t}-P_{D, i}}\right]
$$

Eq. (8) indeed works for both directions.

Fig. 3 (a) plots the model results of a minor loop and the saturation loop (data from [16]). The trace of $\mathrm{C}$ to $\mathrm{B}$ follows a different coercive voltage compared to the trance of $\mathrm{A}$ to $\mathrm{B}$. The reason is that at point $\mathrm{C}$ there are switched downwards domains which impose an additional field upon the unswitched upwards domains and change the overall coercive voltage [21]. Fig.3 (b) plots a smaller minor loop inside another minor loop. The memory wiping-out of turning points [13] applies here.
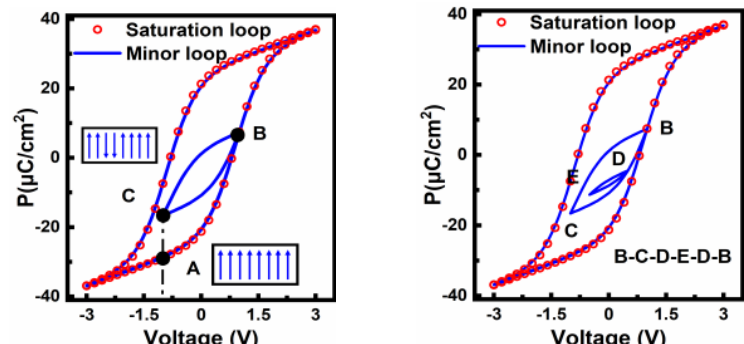

Fig.3. The quasi-static model calculates the minor loop by adjusting the coercive voltage. (a) The physics behind the minor loop is the interaction of the switched domain on the unswitched ones. (b) The turning points are stored and wiped out for the trajectory of minor loops.

\section{A NON-QUASI-STATIC FERROELECTRIC MODEL}

The non-quasi-static (NQS) model is derived with the state transition in the time and voltage domains. From the equations in Fig.1 (a), a differential algebraic equation is derived:

$$
\frac{d P_{D, \mathrm{NQS}}}{d t}+\frac{P_{D, N Q S}}{\tau}=\frac{1}{\tau} \frac{r_{D U}}{r_{D U}+r_{U D}}
$$

in which a time constant is defined. For the case of nucleation limited switching (NLS), the time constant [18] as a function of voltage is given by:

$$
\tau=\frac{1}{r_{U D}+r_{D U}} \cong \tau_{0} \exp \left(\frac{V_{a}}{\left|V_{f e}\right|}\right)^{\alpha}
$$


At the slow limit, $P_{D, N Q S}$ reaches its quasi-static value $P_{D}$. Thus Eq. (9) is re-written:

$$
\frac{d P_{D, N Q S}}{d t}+\frac{P_{D, N Q S}}{\tau}=\frac{P_{D}}{\tau}
$$

Eq. (11) can be understood from the perspective of a relaxation approximation $[22,23]$. The domain switching is only finished with a period, and thus is not responding simultaneously to a faster voltage signal. A deficit in polarization, $P_{D, d e f}=P_{D-} P_{D, N Q S}$, is the driving force to complete the dynamic switching. The current is given by:

$$
i=\frac{P_{D}-P_{D, N Q S}}{\tau} \cdot 2 P_{s}+C_{f e} \frac{d V_{f e}}{d t}
$$
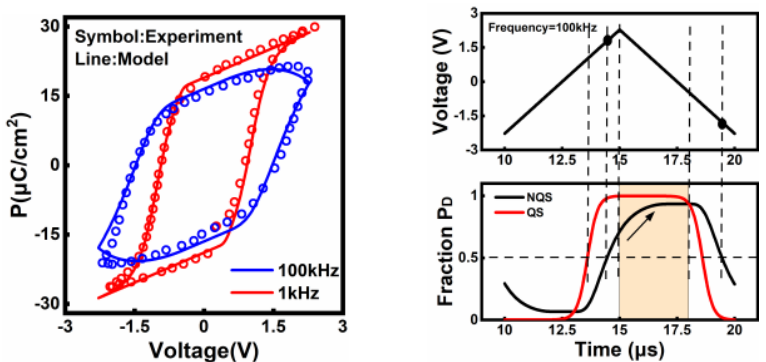

Fig.4. The non-quasi-static model is verified by reproducing the frequency dependent P-V characteristics. (a) Measurements under high frequency show increased coercive voltages and reduced remnant polarization. (b) The relaxation of polarization explains well the frequency dependence.

Fig.4 (a) plots the NQS model result in comparisons to those of measurement data [20]. P-V curves under triangular voltage waveforms of two frequency are shown, and the NQS model agree well with the data. For the case of frequency $f=100 \mathrm{kHz}$, the P-V curve in the voltage domain shows an increased coercive voltage, and a rounded corner (similar to the $\mathrm{P}-\mathrm{V}$ with leakage [24]). Fig.4 (b) plots the dynamic polarization in time domain, correspondingly. $V_{c}$ with higher frequency is defined as the voltage under which $P_{D}$ reaches 0.5 , which indeed comes from the dynamic switching effect. The NQS model calculates the polarization relaxation correctly, as shown by the dashed lines in Fig.4 (b). The rounded corner is also due to the delayed polarization. When the voltage starts retreating from its peak, the time constant is still small allowing further switching of the deficit polarization. As the time constant increases further with reduced voltages, the switching process is 'frozen'. The dashed lines in Fig.4 (b) shows this process, and explains the reduced remnant polarization $P_{r}$, as well as the rounded corner.
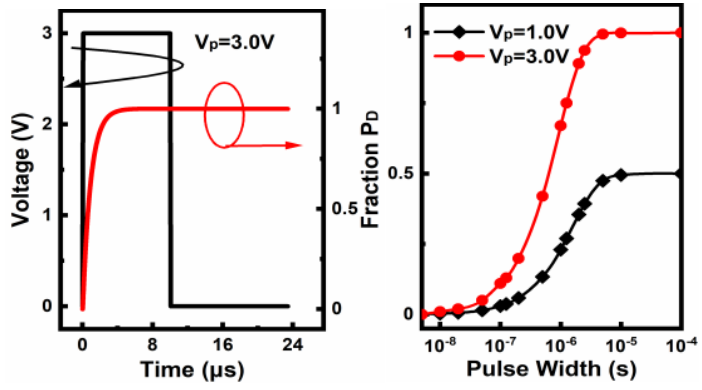

Fig.5. The non-quasi-static model is applied to calculate the pulse width dependent switching. (a) The voltage pulse and the switched polarization in the time domain. (b) The trade-off between pulse amplitude and width in programing the ferroelectric capacitance.
With the switching time parameters from the frequency dependent $\mathrm{P}-\mathrm{V}$ measurement, the pulse programming property of ferroelectric capacitance is calculated consistently. Fig. 5(a) plots the polarization by a voltage pulse in the time domain, and Fig.5 (b) shows the switched fraction with in a pulse. The pulse width dependence is reproduced.

\section{V.CONCLUSION}

In this work, a non-quasi-static model for polarization switching in ferroelectric capacitances is developed. Similar to the Preisach model, a macro polarization is assumed without involving complex domain distributions. Different from the Preisach model, a physics-based minor loop is developed from coercive voltage modulation perspective, and a relaxation in polarization is derived. The formulations developed in the framework of state transitions, reproduce the experimental data of saturation loop, minor loop and frequency dependent $\mathrm{P}-\mathrm{V}$ curves.

\section{REFERENCES}

[1] A.I.Khan,A.Keshavarzi,S.Datta,"The future of ferroelectric field-effect transistor technology," Nature Electronics, vol.3, no.10, pp-588-597, Oct.2020. DOI:10.1038/s41928-020-00492-7

[2] T.Mikolajick,S.Slesazeck,M.H.Parl,U.Schroeder, ” Ferroelectric hafnium oxide for ferroelectric random-access memories and ferroelectric field-effect transistors," MRS Bulletin, vol.43, no.5, pp.340-346, May.2018.DOI: $10.1557 / \mathrm{mrs} .2018 .92$

[3] M. Trentzsch, S. Flachowsky, R. Richter, J. Paul, "A 28nm HKMG super low power embedded NVM technology based on ferroelectric FETs," In IEDM, 2016, pp. 11.5.1-11.5.4, pp. 11.5.1-11.5.4, Dec. 2016. DOI: 10.1109/IEDM.2016.7838397.

[4] K. Ni,P.Sharma,J.Zhang. et al, "Critical Role of Interlayer in $\mathrm{Hf}_{0.5} \mathrm{Zr}_{0.5} \mathrm{O}_{2}$ Ferroelectric FET Nonvolatile Memory Performance," IEEE Trans. Electron Devices, vol. 65, no. 6, pp. 2461-2469, June 2018, DOI: 10.1109/TED.2018.2829122.

[5] T.Ali, K.Kuhnel, M.Czernohorsky, C.Mart.et al, "A Study on the Temperature-Dependent Operation of Fluorite-Structure-Based Ferroelectric $\mathrm{HfO}_{2}$ Memory FeFET: A Temperature-Modulated Operation," IEEE Trans. Electron Devices, vol. 67, no. 7, pp. 2793-2799, July 2020, DOI: 10.1109/TED.2020.2996582.

[6] S.Oh, T.Kim, M.Kwak, et al., " $\mathrm{HfZrO}_{\mathrm{x}}$-Based Ferroelectric Synapse Device With 32 Levels of Conductance States for Neuromorphic Applications," IEEE Electron Dev. Lett.,vol. 38, no. 6, pp. 732-735, June 2017, DOI: 10.1109/LED.2017.2698083.

[7] X. Sun, P. Wang, K. Ni, S. Datta and S. Yu. "Exploiting Hybrid Precision for Training and Inference: A 2T-1FeFET Based Analog Synaptic Weight Cell,,"In IEDM, 2018, pp. 3.1.1-3.1.4, DOI: 10.1109/IEDM.2018.8614611.

[8] J. Luo, L. Yu, T. Liu, et al., "Capacitor-less Stochastic Leaky-FeFET Neuron of Both Excitatory and Inhibitory Connections for SNN with Reduced Hardware Cost," In IEDM, 2019, pp. 6.4.1-6.4.4, DOI: 10.1109/IEDM19573.2019.8993535.

[9] Y. Fang, J. Gomez, Z. Wang, S. Datta, A. I. Khan and A. Raychowdhury, "Neuro-Mimetic Dynamics of a Ferroelectric FET-Based Spiking Neuron," IEEE Electron Dev. Lett., vol. 40, no. 7, pp. 1213-1216, July 2019, DOI: 10.1109/LED.2019.2914882.

[10] S. Mueller, S. R. Summerfelt, J. Muller, U. Schroeder and T. Mikolajick, "Ten-Nanometer Ferroelectric Si:HfO ${ }_{2}$ Films for Next-Generation FRAM Capacitors," IEEE Electron Dev. Lett., vol. 33, no. 9, pp. 1300-1302, Sept. 2012, DOI:10.1109/LED.2012.2204856.

[11] A.I.Khan, K.Chatterjee, B.Wang, et al.,"Negative capacitance in a ferroelectric capacitor," Nature Materials, vol. 14, no. 2, pp. 182-186, Feb. 2015. DOI: 10.1038/NMAT4148 
[12] K. Ni, W. Chakraborty, J. Smith, B. Grisafe and S. Datta, "Fundamental Understanding and Control of Device-to-Device Variation in Deeply Scaled Ferroelectric FETs," 2019 Symposium on VLSI Technology, 2019, pp. T40-T41, DOI:10.23919/VLSIT.2019.8776497.

[13] B, Jiang, P. Zurcher, R. Jones, S. Gillespie and J. Lee, "Computationally Efficient Ferroelectric Capacitor Model For Circuit Simulation," 1997 Symposium on VLSI Technology, 1997, pp. 141-142, DOI: 10.1109/VLSIT.1997.623738.

[14] J. Chow, A. Sheikholeslami, J. S. Cross and S. Masui, "A voltagedependent switching-time (VDST) model of ferroelectric capacitors for low-voltage FeRAM circuits," 2004 Symposium on VLSI Circuits. Digest of Technical Papers, 2004, pp. 448-449, DOI: 10.1109/VLSIC.2004.1346646.

[15] B. Obradovic, T. Rakshit, R. Hatcher, J. A. Kittl and M. S. Rodder, "Ferroelectric Switching Delay as Cause of Negative Capacitance and the Implications to NCFETs," 2018 IEEE Symposium on VLSI Technology, 2018, pp. 51-52, DOI: 10.1109/VLSIT.2018.8510628

[16] K. Ni, M. Jerry, J. A. Smith and S. Datta, "A Circuit Compatible Accurate Compact Model for Ferroelectric-FETs," 2018 IEEE Symposium on VLSI Technology, 2018, pp. 131-132, DOI:10.1109/VLSIT.2018.8510622.

[17] B. Schmithusen, P. Tikhomirov, and E. Lyumkis, "Phase-change memory simulations using analytical phase space model," in Proc. SISPAD, pp. 1-4, 2018. DOI: 10.1109/SISPAD.2008.4648236

[18] M. Vopsaroiu, J. Blackburn, M. G. Cain, and P. M. Weaver, "Thermally activated switching kinetics in second-order phase transisiton ferroelectrics," Phys. Rev. B, vol. 82, pp. 024109, 2010. DOI: 10.1103/PhysRevB.82.024109

[19] K. Lim, K. Kim, S. Hong, K. Lee, "A semi-empirical CAD model of ferroelectric capacitor for circuit simulation," 9th International Symposium on Integrated Ferroelectrics, vol. 17, no. 1-4, pp. 97-104, Mar. 1997. DOI: 10.1080/10584589708012985

[20] X. Lyu, M. Si, X. Sun, M.A. Capano, P. Ye, "Fundamental Properties of Ferroelectric and Anti-Ferroelectric Hafnium Zirconium Oxides: Scaling Limit, Switching Speed and Polarization Density," 2020 International Symposium on VLSI Technology, Systems and Applications (VLSI-TSA), 2020, pp. 145-145, DOI: 10.1109/VLSI-TSA48913.2020.9203666.

[21] R. Smith, S. Seelecke, Z. Ounaies, J. Smith "A free energy model for hysteresis in ferroelectric materials" Journal of Intelligent Material Systems and Structures, vol. 14, no. 11, pp. 719-739, Nov,2003. DOI: 10.1177/1045389X03038841

[22] M. Chan, K. Y. Hui, C. Hu, and P. K. Ko, "A robust and physical BSIM3 non-quasi-static transient and AC small-signal model for circuit simulation," IEEE Trans. Electron Devices, vol. 45, no. 4, pp. 834-841, Apr. 1998. Doi: 10.1109/16.662788.

[23] H. Hu, D. Liu, X. Chen, D. Dong, X. Cui, M. Liu, X. Lin, L. Zhang, M. Chan, "A Compact phase change memory model with dynamic state variables," IEEE Trans. Electron Devices, vol. 67, no. 1, pp. 133-139, Jan. 2020. DOI: 10.1109/TED.2019.2956193

[24] R.Meyer, R. Waser, K. Prume, T. Schmitz, S. Tiedke, "Dynamic leakage current compensation in ferroelectric thin-film capacitor structures," Applied Physics Letters, vol. 86, no. 14,pp.142907, Apr,2005. DOI: $10.1063 / 1.1897425$ 